\title{
RENAL TRANSPLANT RELATED ANAESTHESIA EXPERIENCE, CONSIDERATIONS AND PRACTICE OVERVIEW OF AFIU, RAWALPINDI
}

\author{
Maqsood Ahmad, Rafiq Zafar, Muhammad Akmal \\ Combined Military Hospital Okara/National University of Medical Sciences (NUMS) Pakistan
}

\begin{abstract}
Objective: To describe our experience of renal transplant operations in our institute.

Study Design: Prospective observational study.

Place and Duration of Study: Department of Anaesthesiology, Armed Forces Institute of Urology, Rawalpindi, from Sep 2016 to Sep 2019.

Methodology: A total of 170 living related renal transplants were included in this study. Soda bicarbonate was given intra operatively to recipients according to arterial blood gas results. Blood transfusion was done where indicated.

Results: Out of 340 (100\%) patients, 170 (50\%) were living related donors and 170 (50\%) were transplant recipients General anesthesia was the technique of choice. Three (1.76\%) recipients out of 170 were awaked from anesthesia and postponed. Five $(2.94 \%)$ patients were reopened on the same evening and $2(1.17 \%)$ patients had transplant nephrectomy over a period 1 month. Soda bicarbonate was given to $143(84.1 \%)$ and blood transfusion in $4(2.35 \%)$ transplant recipients where as one patient had anaphylaxis intra-operatively.

Conclusion: Living related kidney transplant is the standard transplant procedure in our institute because of non-availability of cadaveric donor. It's now time that deceased donor program be started in Pakistan due to increased cases of renal failure and decreased living donors.
\end{abstract}

Keywords: Arterial blood gases, Metabolic acidosis, Renal transplant, Sodium bicarbonate.

This is an Open Access article distributed under the terms of the Creative Commons Attribution License (https://creativecommons.org/licenses/by-nc/4.0/), which permits unrestricted use, distribution, and reproduction in any medium, provided the original work is properly cited.

\section{INTRODUCTION}

Kidney transplant has evolved over a period of time as the definite method for patients with end stage kidney disease which is recognized when $85-90 \%$ of kidney function has gone; glomerular filtration ratio (GFR) falls $<15$ and their function is not enough for sustaining life ${ }^{1}$. The manifestations of chronic kidney disease are enormous disturbing all body systems ${ }^{2}$. These effects can be controlled by utilizing renal replacement therapy (RRT) which is recommended thrice per week ${ }^{3}$. RRT has its own complications which can be avoided by renal transplant ${ }^{4,5}$. Both cadaveric and living donors are used for renal transplant but very few cases of deceased donor were done in our center. The success of transplant depends upon selection of good quality living donors and recipients. It is always better to go for early transplant after diagnosis of end stage renal failure as long term dialysis complications are innumerable .

Anaesthetic management of kidney transplant is challenging because of multiple underlying problems due to chronic renal failure as well as long term RRT' Cardiovascular system is invariably involved in them, so cardiovascular system was our main focus in pre

Correspondence: Dr Maqsood Ahmad, Consultant Anaesthetist, Combined Military Hospital, Okara Pakistan

Received: 26 Jun 2020; revised received: 26 Jun 2021; accepted: 29 Jun 2021 anaesthesia evaluation along with comprehensive examination of other system ${ }^{8}$. Intraoperative fluid management, hemodynamic monitoring, and maintenance of blood pressure take priority. Use of soda bicarbonate is controversial and depends upon the metabolic acidosis of recipients which is guided by the ABG sample. The intra operative anaesthetic management of transplant is almost same in various centers as far as intra operative fluid administration and hemodynamic monitoring is concerned. Soda bicarbonate is used in few centers whereas it is discouraged in other which all depends upon their practice of tight vs. non tight control of acid base balance.

\section{METHODOLOGY}

This observational analytical study was started after approval (1/RB/2007/107) of the hospital ethics committee and prospectively we included all transplant patients operated in OR. The sample size calculation was done for observational study design and it was about 400 but our total no of patients were less than this no9. The cancerous, severe heart, lung, liver disease, or active infection (tuberculosis) patients were excluded from the study. For donor nephrectomy ASA 1 and 2 patients were operated mostly in left lateral decubitus position under general anaesthesia whereas most recipients were ASA 3 and ASA 4. Hypertension, ischemic heart disease and diabetes were common in 
recipients. Donor nephrectomies were started first using nalbuphine, propofol, and atracurium through $18 \mathrm{G}$ IV line on arm followed by $7.5 \mathrm{~mm}$ in males $(7 \mathrm{~mm}$ in females) endotracheal tube (ETT) as standard practice. Anaesthesia maintenance was on mixture of oxygen in isoflurane on closed circuit and normal saline $30-50 \mathrm{ml}$ was given to either patient intra-operatively. Mannitol was given before clamping the vessels in 200$250 \mathrm{ml}$ of $20 \%$. Recipients were started once the hilum of donor kidney was visible on dissection with slow induction using nalbuphine, propofol, atracurium and intubation. The problem in these patients was hypertension at induction which sometimes continued intraoperatively. Induction was done through $18 \mathrm{G}$ line opposite to dialysis fistula arm. After induction basiliximab was started slowly and given over one hour. Mannitol was started when graft suturing was started while furosemide was given on opening cross clamping. Monitoring was standard for donor nephrectomies except arterial line and CVP. Invasive blood pressure and CVP monitoring was standard for recipients in addition to standard monitoring. ABG was repeated as per requirement after first sample but 2-3 samples per patient were enough to guide acid base disturbance. Soda bicarbonate was given after getting ABG result and once $\mathrm{HCO} 3$ was found low $(<18 \mathrm{mmol} / \mathrm{l})$. No NSAIDS were given intra-operatively and if transfusion was required then CMV negative blood was used. Data thus collected was analyzed using SPSS-16. Descriptive data was presented in mean \pm SD and qualitative data as frequency and percentages.

\section{RESULTS}

A total of $340(100 \%)$ patients were operated under general anaesthesia, including 170 donor nep- hrectomies and 170 recipients. The blood transfusion of irradiated blood was given in only $4(2.35 \%)$ and soda bicarbonate in $146(85.8 \%)$. Our transplant recipient patients were mostly males 159 (93.5\%) whereas donor nephrectomy was mostly females 115 (67.6\%). Children were $3(1.7 \%)$ in no and kidney donated by mothers. Normal saline $30-50 \mathrm{ml} / \mathrm{kg}$ was standard crystalloid solution for donor as well recipient patients. Bleeding was encountered in $4(2.35 \%)$ of donors but only 2 were transfused post-operatively. No mortality was found in either patient intra-operatively. One of the patients died post-operatively due to sudden cardiac arrest and the precipitating cause was most likely uncontrolled hypertension. Intractable metabolic acidosis was found in one patient whom took almost 12 hours post-transplant to settle and dialysis was the last treatment option. Transplant graft rejection happened in 4 patients. Out of 4 patients 3 were operated for nephrectomy on the same night and one after 1 month. Overall 1 year graft survival rate was $92 \%$ in our institute and for coming years it is yet to be calculated.

\section{DISCUSSION}

Deceased donor program is being highlighted at different levels with motivation of masses for organ donation by utilizing different motivational strategies and it will be materialized soon. Living related donor is the only mode of organ donation till now in our institute under the permission of HOTA (Human organ transplant authority) Pakistan ${ }^{10}$. A living donor KDPI (Kidney donor profile index) was recently proposed to compare the quality of liver donor kidneys in USA but we are not following this ${ }^{11}$. Thorough pre anaesthesia evaluation including 2D echocardiography for both donor and recipient along with otherstandard investi-

Table-I: Demographic data in Mean \pm SD.

\begin{tabular}{|c|c|c|c|c|c|c|}
\hline \multirow{2}{*}{\multicolumn{2}{|c|}{ Serial }} & \multirow{2}{*}{ Total } & \multicolumn{2}{|c|}{ Age (years) } & \multirow{2}{*}{ Mean \pm SD } & \multirow[t]{2}{*}{ Remarks } \\
\hline & & & Min & Max & & \\
\hline Donor & 170 & \multirow{2}{*}{340} & & & \multirow{2}{*}{$39.2 \pm 9.6$} & \\
\hline Recipient & 170 & & 22 & 57 & & \\
\hline \multicolumn{2}{|c|}{ Weight in $\mathrm{Kg}$} & 340 & 8 & 75 & $57.5 \pm 15.7$ & \\
\hline
\end{tabular}

Describing demographic data of patients.

Table-II: Basic data of patients undergoing renal transplant.

\begin{tabular}{l|c|c|c}
\hline Serial & Total & Percentage & Remarks \\
\hline Total cases & 340 & $100 \%$ & All operations \\
\hline Donor Nephrectomy & 170 & $50 \%$ & Donor Only \\
\hline Recipients & 170 & $50 \%$ & Kidney recipient \\
\hline Blood Transfusion & 4 & 2.35 & Irradiated blood \\
\hline Soda Bicarb & 146 & 85.8 & When indicated \\
\hline Males & 159 & 93,5 & Out of 340 \\
\hline Females & 115 & 67.6 & Out of 340 \\
\hline Children & 3 & 1.7 & Recipients only \\
\hline
\end{tabular}

Describing the details and percentages of renal transplant patients. 
gations was the pre requisite for pre anaesthesia evaluation. The donors selected were healthy of ASA 1 or 2 and cardiovascular system of recipient was our main focus during assessment as these patients mostly had various degree cardiovascular involvements ${ }^{12}$. About half the mortality of patients on dialysis is due to heart failure and cardiovascular disease is the leading cause of death (and therefore graft loss) after renal transplantation ${ }^{13}$. Similarly pulmonary function test (PFT's) were done in cases where indicated especially with type 1 diabetes ${ }^{14}$. We have found that few of our patients were taking multiple antihypertensive drugs when they landed in OR (operating room) with poor control of blood pressure. The doses of antihypertensive were reduced to half on operation day as these patients undergo profound hypotension after induction which becomes difficult to treat. Ejection fraction $<35 \%$ recipients were not operated as well as those with massive cardiomegaly. Anaemic patients with $(\mathrm{Hb})$ hemoglobin $<6 \mathrm{gm} / \mathrm{dl}$ were not accepted and their $\mathrm{Hb}$ was built preoperatively with no transfusion of blood in OR. Only CMV (cytomegalovirus) negative blood was used if required in emergency. We used general anaesthesia for both donor and recipient patients but epidural or spinal anesthesia can also be used 15. However regional anaesthesia can be contraindicated in recipients due to uremic platelets dysfunction and residual heparin from dialysis ${ }^{16}$.

Plasma Lyte is the fluid of choice for kidney transplant with better preservation of acid base balance ${ }^{17}$. We used normal saline $30-50 \mathrm{ml} / \mathrm{kg}$ depending upon the losses during operation and pre-operative hydration status. Arterial line before induction and central venous line (CVP) after intubation were standard protocol for recipient. Monitoring was standard along with arterial line, CVP, gas agent monitoring and ABG's. We had to wake up one recipient patient after incision once he developed junctional rhythm following bradycardia which persisted. He was sent to cardiology for furthertesting and was operated after one month with no recurrence of the episode. Anaphyllaxis was encountered in one patient related to methylprednisolone sodium succinate and was treated uneventfully.

The dilemma of using soda bicarbonate is point of argument amonganaesthetist. Article written on this controversy, few authors have recommended a tight metabolic control using soda bicarbonate but we have found that the metabolic control depends on optimum routine of dialysis before operation ${ }^{16}$. Arterial blood gases results were used to guide for soda bicarbonate which was given if bicarbonate was $<18 \mathrm{mmol} / 1$ but not in every case. These patients do present with hyperkalemia which can be avoided with dialysis before operation and avoiding Ringer's lactate solution. Similarly hyperglycemia can be detrimental to renal graft and adding to mortality, so blood glucose (aim 80-110 mg/dl) should be well controlled ${ }^{18,19}$.

Keeping MAP (Mean Arterial Pressure) above $70 \mathrm{~mm} \mathrm{Hg}$ and CVP $>12$ was our intraoperative goal for recipients. Adrenaline in bolus of $25 \mu \mathrm{g}$ for hypotension and nitroglycerine in $5 \mu \mathrm{g}$ boluses was used to control blood pressure with deepening of anaesthesia. Most of inotropes have deleterious effect on renal vasculature decreasing renal blood flow whereas keeping renal blood flow is the requirement of successful transplant ${ }^{20}$. Nalbuphine was used for analgesia and continued post-opertively. We did not observe respiratory depression in any patient. Diuretic (Mannitol) were used; first when graft was starting and furosemide on opening the cross clamp. First dose of immunosuppressant (basiliximab) was given at induction and other drug (corticosteroid) at the time of starting graft with fewer side effects ${ }^{21}$.

Most of complications of renal transplantation occur in post op period and vascular complications lead to early graft rejection ${ }^{22}$. PCA (Patient-controlled analgesia) is recommended for post op pain control with morphine and non-steroidal anti-inflammatory agents and cyclooxygenase- 2 inhibitors are contraindicated. Nerve blocks are also used in few centers whereas chronic pain after transplant is common ${ }^{23,24}$.

\section{CONCLUSION}

Kidney transplant remains the treatment of choice for end stage renal disease providing better quality of life, lower death risk, less restrictions of diet and less cost as compare to dialysis. Preemptive kidney transplant is also utilized for few patients before even starting dialysis. Rationing of dialysis is required to streamline the overburdened staff and equipment because of large no of patients presenting with end stage renal disease. Similarly, speeding up the deceased donor program is the ultimate requirement of developing countries for providing enough supply of kidney for patients on dialysis and renal failure. Governments should take initiative and necessary steps for helping institute to develop curriculum for transplant and guidelines which is although provided by HOTA Pakistan but efforts are meager. 


\section{CONFLICT OF INTEREST}

This study has no conflict of interest to be declared by any author.

\section{REFERENCES}

1. Martin WP, White J, López-Hernández FJ, Docherty NG, le Roux CW. Metabolic surgery to treat obesity in diabetic kidney disease, chronic kidney disease, and end-stage kidney disease; what are the unanswered questions?. Front Endocrinol 2020; 11(1): 289-92.

2. John F. Butterworkth, David C, Mackey, John D, Wasnick. Morgan \& Mikhail 6 ed. Lange, Mc Graw Hill Education; 2019 [Internet] Available at. https://www.mhprofessional.com/ 9781259834424-usa-morgan-and-mikhails-clinicalanesthesiology-6th-edition-group. (Assessed at: Sep 16, 2016)

3. Yelena S, Nancy G, Areeft S, Roderick M, Carin O, Indulis R, et al. Wilth Timing of Dialysis Initiation, Duration and Frequency of Hemodialysis Sessions, and Membrane Flux: A Systematic Review for a KDOQI Clinical Practice Guideline. Am J Kidney Dis 2015; 66(5): 823-36.

4. Craswell A, Massey D, Wallis M, Sriram D, Gray NA, Kotwal S. Current practice in dialysis central venous catheter management: Multi-disciplinary renal team perspectives. Nephrol 2020; 25(5): 406-12.

5. Issa A, Yacoub A, Fady M, Suad H. Interdialytic weight gain in hemodialysis: epidemiology and outcomes, Nephrol Dialysis Transplantat 2020; 35(3): 10-14.

6. Robert T, Abbas K, John R. Chronic kidney disease and its complications. Prim Care; 2008; 35 (2): 4-7.

7. Rudin D, Gentian H, Hektor S, Nehat B, Myzafer, K, Artan B, et al. From pre- existing renal failure to perioperative renal protection: the anesthesiologist 's dilemma. Anesth Pain Med 2016; 6(3): 32386-90.

8. Samantha L, Gelfand A, Blaise A, Brenda H, Amanda K, Leonberg Y. American Journal of kidney disease. 2020; 75(2); 245-55.

9. Sharma SK, Mudgal SK, Thakur K, Gaur R. How to calculate sample size for observational and experimental nursing research studies?. Natl J Physiol Pharm Pharmacol 2020; 10(1): 1-8.

10. Naveed K, Hameed Z, Khalid A, Hamid A, Fatima B, Batool A. Exploratory study on organ donation and organ transplantation in Pakistan. In proceedings of the tenth international conference on information and communication technologies and development. ICPS 2019; 49(1): 1-4.

11. Stoumpos S, Jardine A, Mark P. Cardiovascular morbidity and mortality after kidney transplantation. Transpl Int 2015; 28(1);
$10-21$.

12. Saritas T, Floege J. Cardiovascular disease in patients with chronic kidney disease. Herz 2020; 45(2): 1-7.

13. Shingde R, Calisa V, Craig JC, Chapman JR, Webster AC, Pleass $\mathrm{H}$, et al. Relative survival and quality of life benefits of pancreaskidney transplantation, deceased kidney transplantation and dialysis in type 1 diabetes mellitus-a probabilistic simulation model. Transplant Inter 2020; 33(11): 1393-04.

14. Silva WA, Pinheiro AM, Junior CC, Lima PH, Costa KM, Júnior $\mathrm{JH}$ et al. Anesthesia for renal transplant surgery in adults. J Surg Clin Res 2020; 11(2): 128-34.

15. Escolar G, Díaz-Ricart M, Cases A. Uremic platelet dysfunction ref. Current Hematol Reports 2005; 4(1): 359-67.

16. Gonzalez-Castro A, Ortiz-Lasa M, Peñasco Y, González C, Blanco C, Rodriguez-Borregan JC. Choice of fluids in the perioperative period of kidney transplantation. Nefrología (Eng Edi) 2017; 37(6): 572-78.

17. Parekh J, Niemann CU, Dang K. Intraoperative hyperglycemia augments ischemia reperfusion injury in renal transplantation: a prospective study. J Transplant 2020; 45(2): 122-28.

18. Coemans M, Van Loon E, Lerut E, Gillard P, Sprangers B, Senev A, et. al. Occurrence of diabetic nephropathy after renal transplantation despite intensive glycemic control: an observational cohort study. Diabetes care 2019; 42(4): 625-34.

19. Etezadi F, Abrandabadi AN, Motaharinia J, Mojtahedzadeh M, Pourfakhr P, Khajavi MR, et al. the effect of osmotherapy and tight control of acidosis on early graft function among deceaseddonor kidney transplant recipients: a randomized controlled trial. Int J Organ Transplant Med 2017; 8(1): 8-10.

20. Tönshoff B. Immunosuppressive therapy post-transplantation in children: what the clinician needs to know. Exp Rev Clin Immunol 2020; 16(2): 139-54.

21. Gómez Dos Santos V, Gallego S, FJ BR. The usefulness of contrast-enhanced ultrasound in the assessment of early kidney transplant function and complications. Diagnostics (Basel, Switzerland) 2017; 7(3): 53-60.

22. Shoeibi G, Babakhani B, Mohammadi SS. The efficacy of ilioinguinaliliohypogastric and intercostals nerve co-blockage for postoperative pain relief in kidney recipients. Anesth Analg 2009; 108(1): 330-33.

23. Mukhtar K, Khattak I. Transversus abdominis plane block for renal transplant recipients. Br J Anaesth 2010; 104(5): 663-64.

24. Jiang $X$, Orton M, Feng R, Hossain E, Malhotra NR, Zager EL, et al. Chronic opioid usage in surgical patients in a large academic center. Annals Surg 2017; 265(4): 722-25. 